\title{
Grinfeld instability on crack surfaces
}

\author{
R. Spatschek and Efim A. Brener \\ Institut für Festkörperforschung, Forschungszentrum Jülich, D-52425 Jülich, Germany
}

(Received 1 February 2001; published 24 September 2001)

\begin{abstract}
The surface of a propagating crack is shown to be morphologically unstable because of the nonhydrostatic stresses near the surface (Asaro-Tiller-Grinfeld instability). We find numerically that the energy of a wavy crack becomes smaller than the energy of a straight crack if the crack length exceeds a critical length $L_{c}$ $=5.18 L_{G}\left(L_{G}\right.$ is the Griffith length). We analyze the dynamic evolution of this instability, governed by surface diffusion or condensation and evaporation. It turns out that the curvature of the crack surface becomes divergent near the crack tips. This implies that the widely used condition of the disappearance of $K_{I I}$, the stress intensity factor of the sliding mode, is replaced by the more general requirement of matching chemical potentials of the crack surfaces at the tips. The results are generalized to situations of different external loading.

DOI: 10.1103/PhysRevE.64.046120

PACS number(s): 62.20.Mk, 46.50.+a, 81.40.Np
\end{abstract}

\section{INTRODUCTION}

The uniform motion of a straight crack is well understood [1]: A crack exceeding a certain critical length, the Griffith length, starts to grow, since the energy gain due to elastic relaxation is bigger than the loss of surface energy that appears as a consequence of the elongation of crack surfaces.

However, in experiments the surfaces of a crack are often rough [2]. Some of these results are interpreted in the framework of models of cracks propagating in heterogeneous media. The other possibility for the roughening of the crack surfaces is the instability of the straight motion of the crack tip. Recent experiments revealed that many puzzling phenomena in brittle fracture are related to an oscillatory instability at velocities appreciably below the Rayleigh speed [3]. There were several attempts in literature to investigate the stability of a propagating crack. The linear stability analysis of the quasistatic crack subject to mode I (opening mode) loading has been performed by Cotterell and Rice [4] with subsequent refinement by Adda-Bedia and Ben Amar [5]. They employ the Griffith theory and the so-called principle of local symmetry, i.e., the condition that mode II (sliding mode) stress intensity factor $K_{I I}$ vanishes at the tip of the crack. They found that the straight motion of the crack becomes unstable if the tangential loading exceeds a critical value.

A full dynamical model, including the microscopic description of the cohesive zone around the crack tip, has been developed by Ching, Langer, and Nakanishi [6]. The cohesive force in the neighborhood of the tip provides a fracture energy and a mechanism for regularizing the stress singularity; this model removes the need to speculate about a principle of local symmetry. In addition to Refs. [4,5], they found a strong microscopic instability even for very low crack velocities, which depends very sensitively on tiny details of the cohesive-zone model. However, later Langer and Lobkovsky [7] showed that these cohesive-zone models in the framework of a sharp-tip representation lead to unphysically unreasonable features of the elastic stresses in spite of a regularization by cohesive forces.

We strongly point out that in all these descriptions a crack is recognized as the trace left behind by the propagating crack tip. In this sense its surfaces are "frozen" and not subject to any additional dynamics.

On the other hand, another elastic instability has attracted much interest in the recent time: Grinfeld [8] and Asaro and Tiller [8] discovered that the energy of a non-hydrostatically stressed solid with a flat surface can be diminished by a change of its shape and formation of deep grooves [9]. This deformation is not due to elastic strain but to redistribution of matter along the surface. Apart from surface diffusion other transport mechanisms can be taken into account as well: For example, a solid phase that is in contact with its melt grows due to melting and recrystallization. Similarly an evaporation-condensation mechanism is also conceivable.

In all cases the central reason for this instability is again a drastic decrease of elastic energy during the deformation process. This decrease is bigger than the accompanying increase of surface energy for relatively long-wave interface perturbations. Grinfeld performed a lowest order stability analysis where he described the temporal evolution of a curved interface shape $y(x, t)=y_{0} \exp (i k x+\lambda t)$. The line $y=0$ corresponds to the initial unperturbed interface of a twodimensional body in the $x-y$ plane.

In the case of surface diffusion the time-evolution is governed by the dispersion relation

$$
\lambda=D v_{s} k^{2}\left[\frac{2 \sigma_{0}^{2}\left(1-\nu^{2}\right)}{E}|k|-\alpha k^{2}\right],
$$

where $D$ is proportional to the surface diffusivity and $v_{s}$ the atomic volume. $E$ and $\nu$ are the Young and Poisson coefficients respectively, $\alpha$ is the surface tension. $\sigma_{0}=\sigma_{n n}$ $-\sigma_{\tau \tau}$, with normal and tangent directions $n$ and $\tau$, reflects the nonhydrostaticity of the stress tensor $\sigma$. Here one can easily see that long-wave perturbations lead to the Grinfeld instability, whereas short-wave perturbations are hampered by surface tension. The most unstable mode $k_{m}=3 \sigma_{0}^{2}(1$ $\left.-\nu^{2}\right) / 2 \alpha E$ evolves with $\lambda_{m}=D v_{s} \alpha k_{m}^{4} / 3$.

Recently, it was emphasized in Ref. [10] that the condition of nonvanishing $\sigma_{0}$ is fulfilled on the cut interfaces of a straight crack that is loaded perpendicular to the crack at infinity. In this sense a straight crack cannot be a stable configuration under all circumstances, because slight deforma- 


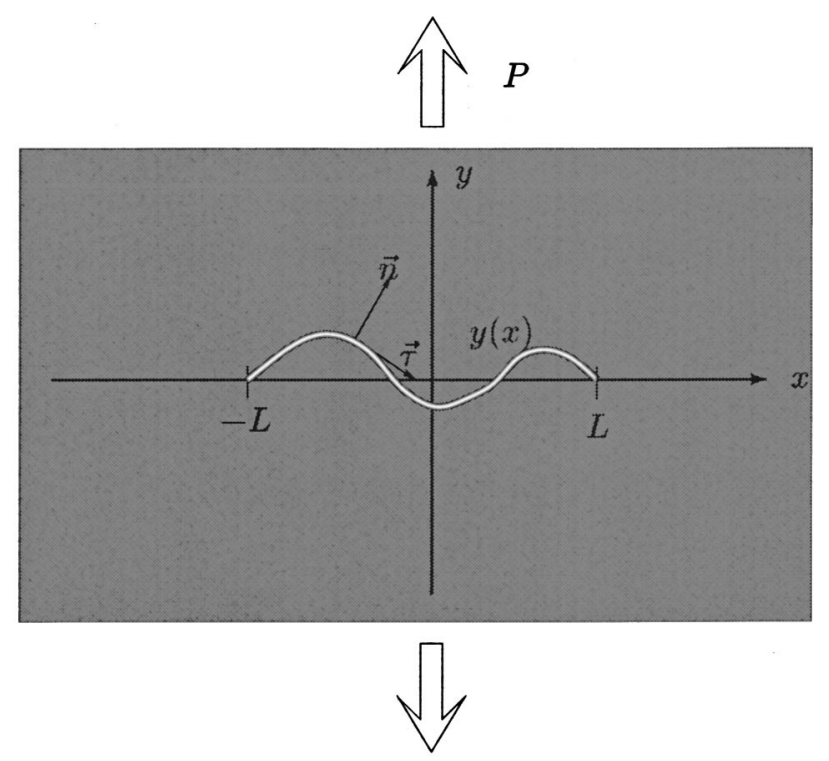

FIG. 1. Geometry of a wavy crack in a two-dimensional solid.

tions may reduce the total energy. In Ref. [10] it was predicted that this can happen provided that a certain critical crack length is exceeded.

The aim of the current paper is a deeper understanding of this instability. It is organized as follows: In Sec. II we derive expressions to compare the energy of a static straight and a wavy crack. In Sec. III the dynamics of crack deformation beyond the threshold of instability is analyzed. A more detailed investigation of the behavior near the singular crack tips is performed, and the situation is generalized to not necessarily parallel crack surfaces. In Sec. IV we consider different loading mode situations. Appendix A contains a solution of the elastic problem of a crack with independent surfaces. Appendix B is a proof for the equivalence of two representations of the elastic energy that are derived in Sec. II.

\section{STABILITY ANALYSIS}

As mentioned above a crack with a length different from the Griffith length wants either to grow until the whole material is fractured into pieces or to shrink until it disappears completely. In order to study the quasistatic kinetics of the Grinfeld instability, this fast straight motion must be suppressed. Formally we fix the tip positions of the crack and only discuss shape deformations.

The key question is whether a straight crack is stable with respect to small perturbations of its shape.

For the moment we restrict our considerations to the case of parallel crack surfaces (mathematical crack). Thus we can describe the crack shape by a function $y(x)$, with $-L<x$ $<L$ (see Fig. 1). The tips are located at $x= \pm L, y=0$ and the straight crack corresponds to the shape function $y(x)$ $\equiv 0$. The goal of this section is to derive expressions for the energy change due to shape deformations $U[y]-U[0]$. This functional depends in a complicated, nonlocal way on the function $y(x)$. We normalize $U[0]=0$. Since the homoge-

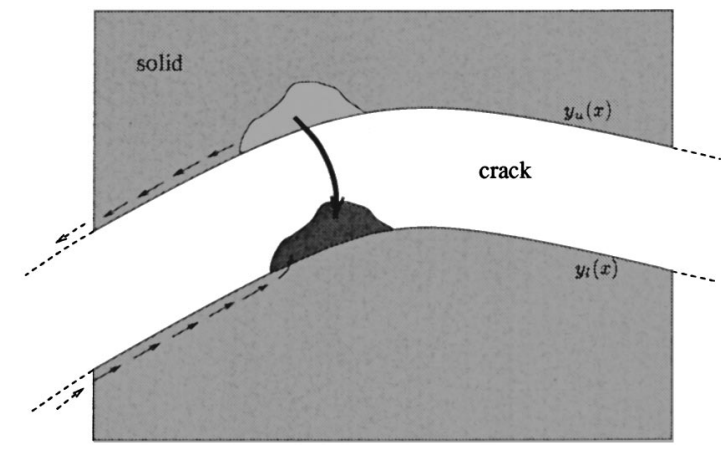

FIG. 2. Deformation of the crack shape as a result of a reshuffling of matter. This can happen either directly through the crack or along the crack surfaces and around the tips.

neous external stress $\sigma_{y y}^{\infty}=P$ acts perpendicular to the straight crack, it is clear from symmetry that two cracks $y(x)$ and $-y(x)$ must have the same energy. Thus $O\left(y^{2}\right)$ is the lowest nonvanishing contribution to $U[y]$.

From now on we assume the special case of a twodimensional plane-strain situation.

The basic idea to derive the change of energy during deformation of the crack shape is founded on the expression for the chemical potential of the solid phase at an interface [11]

$$
\mu_{s}=v_{s}\left[f_{s 0}+\frac{1-\nu^{2}}{2 E}\left(\sigma_{\tau \tau}-\sigma_{n n}\right)^{2}+\alpha \kappa\right] .
$$

Here $v_{s}$ is the atomic volume of the solid phase; $f_{s 0}$ is the free energy density for a hydrostatic situation; $\alpha$ is the surface energy; $\kappa$ is the curvature of the interface (counted positive for a convex solid).

In principle one has to remove matter from one front of the crack and deposit it at the opposite one. In this way the originally straight crack is deformed to its final wavy shape. This procedure is outlined in Fig. 2.

Later different mechanisms for this transport process will be discussed: The removed matter can either cross directly through the interior of the crack or diffuse along the surfaces. The first case corresponds to a evaporation-condensation process; in the second case of surface diffusion the matter must wander around the crack tips. For energetic considerations the precise transport process is of course irrelevant; it becomes important later for dynamical approaches.

An easy way to calculate the energy of a perturbed crack was proposed in Ref. [10]. The total energy change consists of a change of both the surface energy and the elastic energy.

First a deformation increases the arc length of the crack and therefore the surface energy. To the lowest nonvanishing order this change is given by

$$
U_{s}=2 \alpha \int_{-L}^{L} \frac{\left(y^{\prime}\right)^{2}}{2} d x
$$

The factor 2 appears because the crack consists of two interfaces.

Additionally, the change of geometry also alters the stored elastic energy. We calculate this energy change in two 
steps: First we keep the stress field of the straight crack artificially fixed in the whole solid while the material is reshuffled along the two sides of the crack. One uses the expression for the chemical potential (2) to calculate the required energy. When the final shape is reached, the usual condition of vanishing normal and shear stress along the crack surfaces, $\sigma_{n n}=\sigma_{n \tau}=0$, is clearly violated. Therefore one has to adjust the stress appropriately by adding a compensation field $\sigma^{(1)}$ and book keeping the relaxation of energy.

Let us begin with the first contribution to the elastic energy. The analytic continuation of the stress field in the vicinity of a straight crack up to first order is given by (see for example [12])

$$
\begin{aligned}
& \sigma_{x x}=-P\left[1 \mp \frac{2 L^{2} y}{\left(L^{2}-x^{2}\right)^{3 / 2}}\right]+O\left(y^{2}\right), \\
& \sigma_{y y}=0+O\left(y^{2}\right), \\
& \sigma_{x y}=0+O\left(y^{2}\right) .
\end{aligned}
$$

The minus branch in $\sigma_{x x}$ corresponds to the upper, the plus branch to the lower crack surface. Therefore the elastic contribution to the chemical potential (2) at the upper crack surface is (the shape-independent parts are irrelevant and therefore neglected)

$$
\left[\mu_{s}\right]_{u}=-\frac{2 v_{s} P^{2}\left(1-v^{2}\right) L^{2}}{E\left(L^{2}-x^{2}\right)^{3 / 2}} y .
$$

At the lower interface $\left[\mu_{s}\right]_{l}=-\left[\mu_{s}\right]_{u}$. We note that due to the fixing of the stress field the chemical potential is also fixed during the redistribution of matter. It results in

$$
\begin{aligned}
U_{e l s} & =-2 \int_{-L}^{L} \int_{y=0}^{y(x)} \frac{\left[\mu_{s}\right]_{u}}{v_{s}} d y d x \\
& =\int_{-L}^{L} d x \frac{4 P^{2}\left(1-\nu^{2}\right) L^{2}}{E\left(L^{2}-x^{2}\right)^{3 / 2}} \frac{y^{2}}{2} \\
& =-\frac{2 P^{2}\left(1-\nu^{2}\right)}{E} \int_{-L}^{L} d x\left(y^{2}\right)^{\prime \prime} \sqrt{L^{2}-x^{2}} .
\end{aligned}
$$

As one can see from the second representation this energy contribution is always positive, i.e., stabilizing. The last representation results from integration by parts which requires the boundary conditions $y( \pm L)=0$ and corresponds to fixed crack tips.

The second contribution to the change of the elastic energy comes from the adjustment of the stress field. From Eq. (4) it follows that after performing the first step the shear stress along the new crack surface $y(x)$ is $\sigma_{n \tau}=P y^{\prime}$ $+O\left(y^{2}\right)$. To this order the normal component is already correct: $\sigma_{n n}=0$. The compensation field $\sigma^{(1)}$ introduced above must cancel these boundary values and vanish at infinity. It corresponds to the stress field of a straight crack with surface tractions $T_{s}=-P y^{\prime}$ and $T_{n}=0$.
We imagine to create this crack by cutting the solid, starting at the left tip $x=-L$ and proceeding to $x=+L$. The associated mode II stress intensity factor $K_{I I}$ is calculated in [4]. Assume that the current crack tip position is $x=L^{\prime}$ with $-L<L^{\prime}<L$, it is given by

$$
K_{I I}\left(L^{\prime}\right)=\sqrt{\frac{2}{\pi\left(L^{\prime}+L\right)} P} \int_{x=-L}^{L^{\prime}} \sqrt{\frac{L+x}{L^{\prime}-x}} y^{\prime}(x) d x .
$$

The energy release rate coming along with an increase of the crack length by $d L^{\prime}$ is given by Irwin's formula [13]

$$
U_{e l u}=-\frac{1-\nu^{2}}{E} \int_{-L}^{L} K_{I I}^{2}\left(L^{\prime}\right) d L^{\prime} .
$$

Finally the total energy of the wavy crack is given by the sum

$$
U[y]=U_{s}+U_{e l s}+U_{e l u} .
$$

Alternatively, and more intuitively, the total energy of the crack can be calculated as follows: As before one can imagine to reshuffle the matter along the crack surfaces to obtain the final wavy shape, but this time the stress field is not fixed during the redistribution. At each step during this process the condition of vanishing normal and shear stress at the crack surfaces must be fulfilled. This requires the solution of the elastic problem of a wavy crack; its solution is derived in Appendix A for the generalized situation of not necessarily parallel crack surfaces. Inserting these expressions (A14)(A16) into the chemical potential (2) gives to first order in $y$

$$
\begin{aligned}
{\left[\mu_{s}\right]_{u / l}=} & v_{s}\left[f_{s 0}+\frac{1-v^{2}}{2 E}\left\{P^{2} \mp \frac{4 P^{2}}{\pi\left(L^{2}-x^{2}\right)^{1 / 2}}\right.\right. \\
& \times \mathcal{P} \int_{-L}^{L} \frac{y^{\prime}(t) \sqrt{L^{2}-t^{2}}}{t-x} d t \\
& \left.\left.\mp \frac{4 P^{2} L^{2}}{\left(L^{2}-x^{2}\right)^{3 / 2}} y(x)\right\} \pm \alpha y^{\prime \prime}(x)\right]
\end{aligned}
$$

( $\mathcal{P}$ denotes the principal value of the integral). In contrast to the former approach, the stress distribution changes during the rearrangement, and therefore the chemical potential (10) depends on the "intermediate shape" $y(x)$. The total energy can now be obtained by integration

$$
U=-\int_{-L}^{L} \int_{y=0}^{y(x)} \frac{\left[\mu_{s}(x)\right]_{u}-\left[\mu_{s}(x)\right]_{l}}{v_{s}} d y d x,
$$

similar to Eq. (6). This results in a completely different representation of $U_{\text {elu }}$.

Though it is clear from physical reasons that both approaches should give the same result, this is not directly visible from the expressions. In particular, two cracks $y(x)$ and $y(-x)$ should have exactly the same energy. Integrating the chemical potential (10) clearly reflects this situation. Also $U_{s}$ and $U_{e l s}$ obey this symmetry but this property is 
less clear for $U_{\text {elu }}$. Since we started to cut the solid at its left tip for derivation of $U_{\text {elu }}$, the resulting formulas (7) and (8) seem to violate this parity invariance at a first glance. Nevertheless it is possible to give a proof of the equivalence of these two different approaches to calculate the energy of the system. It is sketched in Appendix B. The idea is to rederive the expression for the chemical potential (2) from the total energy (9), which proves the equivalence of both ways. Consequently the symmetry condition is also fulfilled, or, in other words, the energy operator $U$ commutes with the parity operator $\hat{P}$, which is defined by $(\hat{P} y)(x)=y(-x)$.

\section{A. Numerical results}

Based on the energy expressions derived in the previous section we are now able to perform a full stability analysis of the problem. We remind that we assigned $U=0$ to the straight crack and hence are interested in the occurrence of $U[y]<0$ for a wavy crack shape. One can easily check that all parameters of the problem appear only in the combination $L_{G}=2 E \alpha / \pi\left(1-\nu^{2}\right) P^{2}$ (Griffith length) in the total energy (9), apart from common prefactors. Thus the minimum energy with respect to all possible crack shapes with a certain length $L$ is simply a function of one single parameter $L / L_{G}$, which easily allows to trace the threshold of instability.

In Ref. [10] minimization has been performed by a variational procedure using only a limited set of analytical representations of shape functions. Here we solve the problem numerically and find the real shape without such restrictions by a full minimization procedure. For that we have chosen a Fourier representation of the crack function $y(x)$, $x \in[-L, L]$

$$
y(x)=\sum_{k=1}^{\infty} b_{k} \sin \frac{k \pi(x+L)}{2 L},
$$

where the upper summation limit is replaced by a sufficiently large cutoff $K$. Since this is linear in the unknown coefficients $b_{k}$ and the total energy is quadratic in the amplitude $y(x)$, we can write the total energy as quadratic form $U$ $=D_{i k} b_{i} b_{k}$ with a real, symmetric $K \times K$ matrix $D$ that depends only on $L / L_{G}$. This matrix can be computed almost completely analytically.

In order to find the minimum of the free energy $U$, a normalization condition is needed, since the amplitude is not restricted in our lowest order calculation. The choice $b_{k} b_{k}$ $=L_{G}^{2}$ is convenient but arbitrary. In this case the minimum of energy is exactly $\lambda_{s} L_{G}^{2}$, where $\lambda_{s}$ is the smallest eigenvalue of the matrix $D$.

It must be remarked that the threshold of instability $U(L)=0$ is not affected by the specific choice of normalization: Minimization requires

$$
\frac{\delta}{\delta y(x)}(U[y]-\tilde{\lambda} f[y])=0,
$$

where $f[y]=0$ is the arbitrary normalization condition, coupled to the energy by the Lagrange multiplier $\tilde{\lambda}$. By construction this is equivalent to

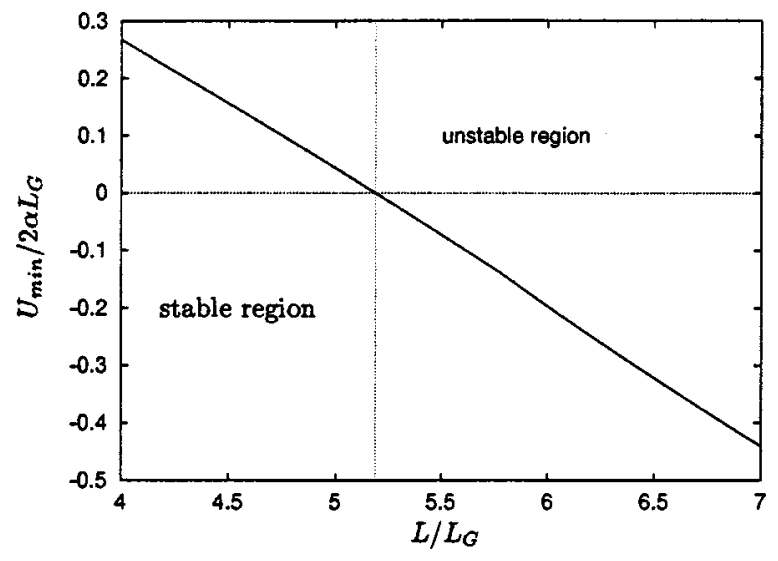

FIG. 3. Minimum normalized energy of a curved crack.

$$
-\frac{2}{v_{s}}\left[\mu_{s}\right]_{u}-\tilde{\lambda} \frac{\delta f}{\delta y}=0,
$$

or, by multiplication with $y(x)$ and integration

$$
\tilde{\lambda}=\frac{U}{\int_{-L}^{L} \frac{\delta f}{\delta y} \frac{y(x)}{2} d x} .
$$

Thus for the threshold of instability, characterized by $U=0$, we have $\tilde{\lambda}=0$ and the normalization condition becomes irrelevant in Eq. (13). Consequently the critical length of the crack and its corresponding shape are universal. For any other length the results depend on the normalization condition. Later the physical meaning of the condition chosen here will become more obvious.

The concrete normalization condition given above is equivalent to

$$
\int_{-L}^{L} y^{2}(x) d x=L L_{G}^{2}
$$

Therefore Eq. (15) reads

$$
\tilde{\lambda}=\frac{U}{L L_{G}^{2}}
$$

and the minimization condition is equivalent to

$$
\mp \frac{2}{v_{s}}\left[\mu_{s}\right]_{u / l}-2 \tilde{\lambda} y(x)=0 .
$$

Figure 3 shows the minimum energy versus crack length. For $L>5.188 L_{G}$ the straight crack becomes unstable and favors a wavy shape. The critical shape is plotted in Fig. 4.

All results turn out to be very robust, and already $K$ $\approx 20$ harmonics are sufficient to describe the shape quite accurately. The code has been checked very carefully against analytically known energy values for special shapes. The result is consistent with the prediction $L_{c}<6 L_{G}$ in Ref. [10].

As we have already seen, the energy operator commutes with the parity operator $\hat{P}$ and therefore all eigenfunctions 


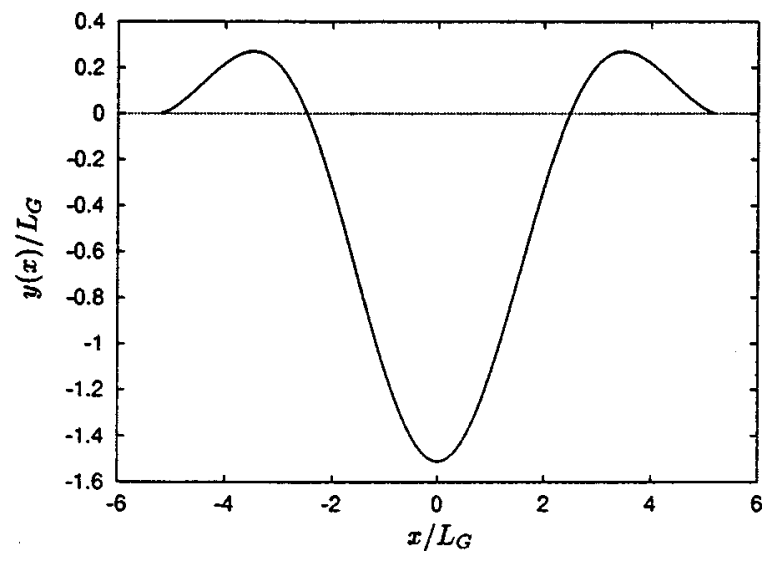

FIG. 4. Universal shape of the critical crack, $L=5.1882 L_{G}$.

are either even or odd. It turns out that the most unstable mode, which belongs to the smallest eigenvalue, is an even function. Thus only terms with odd $k$ appear in the representation (12). Yet there are still certain length intervals where the optimum solution is an odd function.

\section{DYNAMICS OF GRINFELD INSTABILITY}

In this section we go beyond the previous static description where we used the energy to judge whether a certain configuration is stable or not. Here we analyze how a given shape develops in time. If it decays to the straight crack, the crack is stable; otherwise a perturbation develops further and further. This allows to calculate the threshold of instability in a different way and to compare the results with the predictions of the previous section. Again we calculate the chemical potential only up to first order (which corresponds to a quadratic energy). In this sense we cannot expect to obtain new results about the long-time behavior of unstable solutions. Especially we cannot describe the known groovelike structures that are governed by nonlinear effects [9]. Nevertheless this approach is useful because it allows to study more carefully the behavior near the crack tips. This region cannot be described by the previous static approach since the Fourier representation produces strong oscillations there. Instead, we use a real space representation here. By concentrating more grid points in the sensitive tip region we are able to study the peculiarities occurring there quite accurately.

Again we start with the special case of coherent surfaces $y_{u}(x)=y_{l}(x)$. Later we will generalize this situation.

Redistribution of matter is driven by spatial variations of the chemical potential of the solid phase along the crack surfaces. We analyze two different transport mechanisms in this section: Surface diffusion is described by the equation

$$
\frac{\partial y(x, t)}{\partial t}=-D \nabla^{2} \mu_{s}(x, t)
$$

From the representation of the chemical potential, explicitly given in Eq. (10), it is clear, that Eq. (18) is of fourth order with respect to the spatial derivatives. Basically we are in- terested in a stability analysis, and therefore we look for eigenfunctions of this equation, $y(x, t)=y(x) \exp (\lambda t)$. This leads to

$$
\lambda y(x)=-D \nabla^{2} \hat{\mu}_{s}[y],
$$

where we write $\hat{\mu}_{s}$ for the linear operator of chemical potential that depends nonlocally on the shape $y(x)$.

A simpler dynamics is described by the equation

$$
\frac{\partial y(x, t)}{\partial t}=D_{e c} \mu_{s}(x, t)
$$

with the kinetic coefficient $D_{e c}$. It corresponds to a direct transport of matter through the void of the crack. It is similar to evaporation-condensation (EC) processes known, e.g., from the theory of phase separation. We mainly introduce this mechanism because of its simplicity that is useful for testing purposes of the numerical code.

In both languages the threshold of instability corresponds to $\lambda=0$ and should be the same. As before, the equations of motion depend only on the single adjustable parameter $L / L_{G}$. Thus the different eigenvalues are also only a function of this parameter, and by simple plotting one can easily detect the crossing point $\lambda\left(L_{c} / L_{G}\right)=0$.

Both mechanisms are purely dissipative and we expect that all eigenvalues are real. One can readily check that the operator $\hat{\mu}_{s}$ fulfills the self-adjointness condition $\left(\hat{\mu}_{s} y_{1}, y_{2}\right)$ $=\left(y_{1}, \hat{\mu}_{s} y_{2}\right)$ with respect to the standard scalar product $\left(y_{1}, y_{2}\right)=\int_{-L}^{L} \overline{y_{1}(x)} y_{2}(x) d x$. Therefore at least the second mechanism allows only real lambdas.

For the diffusion process we note that the operator $-\nabla^{2}$ is positive definite; one can prove that under these circumstances the compound operator $-\nabla^{2} \hat{\mu}_{s}$ indeed has only real eigenvalues [14].

Since the eigenvalue equation for the meltingrecrystallization mechanism is a second order ordinary integro-differential equation, we require two boundary conditions. As before we demand $y( \pm L)=0$. Surface diffusion requires two additional boundary conditions. Since the chemical potential on the upper and lower surface of the crack [apart from the trivial constant contributions in Eq. (10) that we ignore from now on] is the same but with opposite sign, the condition of a unique value of $\mu_{s}$ requires $\mu_{s}( \pm L)=0$. Otherwise a fast redistribution of matter, driven by the force difference $\left[\mu_{s}\right]_{u}( \pm L)-\left[\mu_{s}\right]_{l}( \pm L)$ would take place in the microscopic region around the crack tips, until the above condition is satisfied. In both cases a critical crack is characterized by the condition $\mu_{s}(x) \equiv 0$.

We use the notation $Y=\left(y, y^{\prime}, \mu_{s}, \mu_{s}^{\prime}\right)$ and discretize these functions on the interval $[-L, L]$. Then the linear equation (19), together with the boundary conditions, can be expressed as $A \cdot Y=0$ with a real, quadratic matrix $A$ that depends on the control parameter $L / L_{G}$ and on the eigenvalue $\lambda$. Since this equation is nonlocal the matrix is not sparse; nontrivial solutions correspond to the condition $\operatorname{det} A=0$, and we use a standard matrix decomposition to 


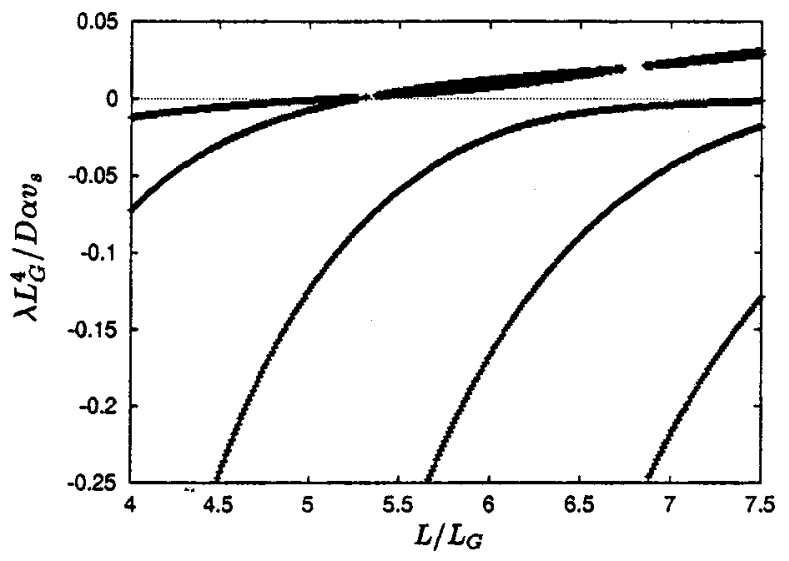

FIG. 5. Eigenvalues of the surface diffusion vs crack length. We detect nontrivial solutions through a change of sign of the determinant. Whenever two curves intersect or come close to each other, this sign does not change. This leads to missing points in the diagram.

detect it [15]: $A=Q \cdot R$, where $R$ is an upper triangular matrix, $Q$ is orthogonal and positive definite, and thus $\operatorname{det} A$ $=\Pi_{i} R_{i i}$.

The allowed eigenvalues as a function of the crack length are shown in Figs. 5 and 6.

To each length belongs an infinite number of eigenfunctions with different eigenvalues; here only the biggest eigenvalues near the threshold of instability are visible. A closer inspection shows that the first crossing of the $\lambda=0$ axis happens at $L_{c}=5.187 L_{G}$, which is in excellent agreement with the previous, static prediction.

Some of the shape functions are illustrated in Fig. 7.

All eigenfunctions are even or odd, but it turns out that not always the even function is the most unstable one. One can clearly see that the two biggest eigenvalue functions of surface diffusion intersect at around $L \approx 5.3 L_{G}$ and again at $L \approx 6.8 L_{G}$; in between the odd branch is the most unstable one. This phenomenon occurs again at bigger crack lengths and also for the EC mechanism.

In both cases the most unstable modes are functions that consist of only one-half or one full period. With descending eigenvalue the number of nodes increases. In this sense the

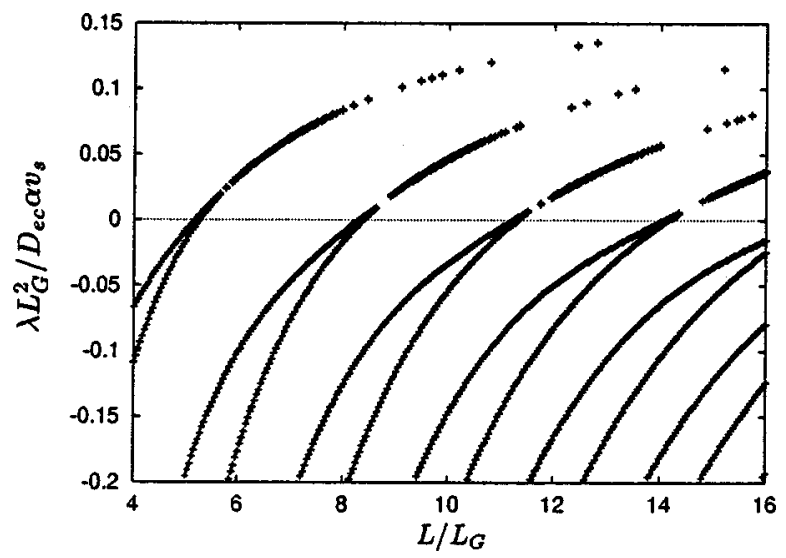

FIG. 6. The same relation for the EC mechanism.
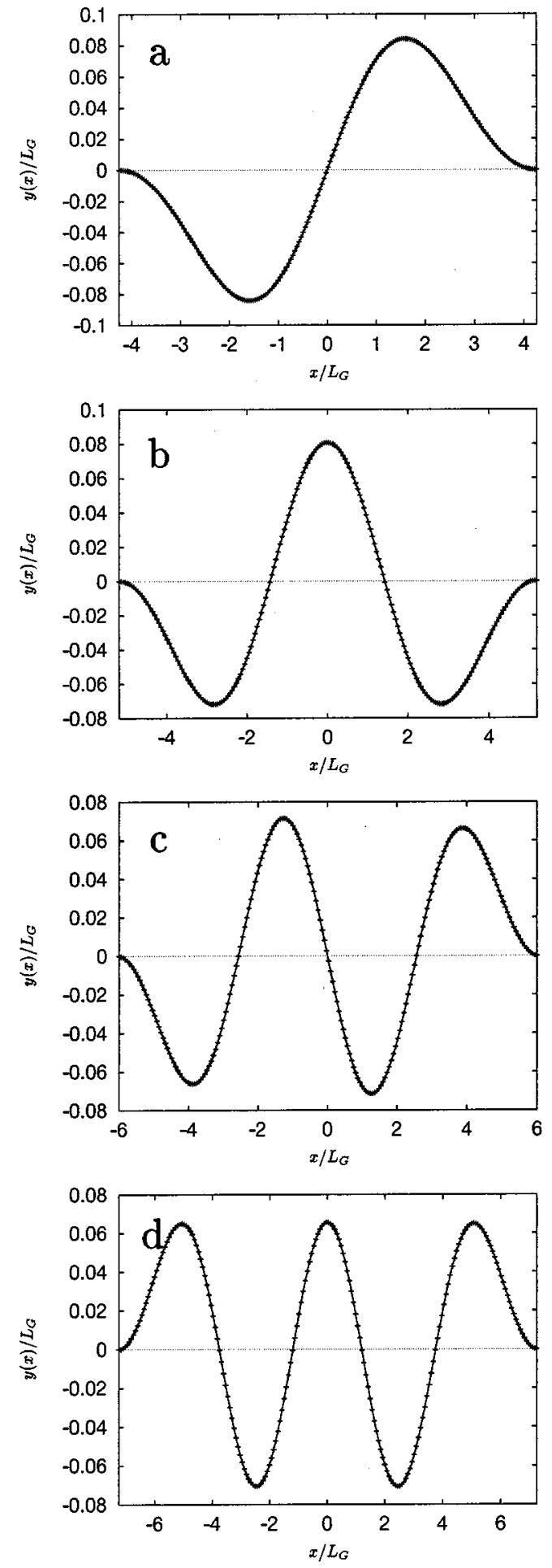

FIG. 7. Some eigenfunctions of surface diffusion. (a) $L$ $=4.26 L_{G}, \lambda=-0.047, \quad$ (b) $L=5.18 L_{G}, \lambda=-0.096, \quad$ (c) $L$ $=6.00 L_{G}, \lambda=-0.167$, (d) $L=7.25 L_{G}, \lambda=-0.167 . \lambda$ is given in units of $D \alpha v_{s} / L_{G}^{4}$.

eigenvalue problem is comparable to simple quantum mechanical eigenstates, e.g., of a single particle in a box.

It is also instructive to use a simplified scaling analysis of the situation: The dispersion relation (1) of the free interface defines a characteristic wavelength $L_{c} \sim k_{c}^{-1} \sim E \alpha /(1$ 
$\left.-\nu^{2}\right) \sigma_{0}^{2}$. The tangential stress at the crack surfaces is $\sigma_{\tau \tau}$ $=\sigma_{0} \sim P$. Thus $L_{c} \sim L_{G}$ which underlines the fact that Grinfeld instability and the Griffith condition for crack growth are basically of the same origin, namely, the competition between surface and elastic energy. Since the tip positions are fixed, only certain perturbation waves fit into this interval. In particular, a minimum crack length $L \sim L_{G}$ must be exceeded to allow at least for one unstable mode.

For the critical crack with $L=L_{c}$, the shape is indistinguishable from the static picture 4 for both mechanisms. We note that also for other lengths the shape functions of the EC mechanism are very similar to those of the diffusion mechanism.

If one plugs in the ansatz $y(x, t)=y(x) \exp (\lambda t)$ into the equation of motion (20), one immediately arrives at the condition (17) for the minimum of the static energy of the last section with the amplitude constraint applied there. Now we see that this arbitrarily chosen constraint is related to the EC mechanism if we identify

$$
\lambda=-\frac{U v_{s} D_{e c}}{L L_{G}^{2}}
$$

with the energy $U$ of the (normalized) crack. First, it is clear from this equation that a stable crack in the static sense with $U>0$ is also stable in the dynamic sense, $\lambda<0$, and vice versa. Furthermore, the lower the crack energy for a given length, the faster the instability develops. The relation (21) also holds numerically: Mapping the two graphs Figs. 3 and 6 using Eq. (21), lets the energy curve exactly conceal the curve of the most unstable eigenvalue.

In the case of very long cracks the spectrum becomes more and more continuous and finally coincides with the spectrum (1), since the boundary conditions become less important. All eigenvalues that are smaller than the maximum value of the Grinfeld spectrum, $\lambda \leqslant \lambda_{m}$, lead to possible solutions. We indeed observe a very good agreement with this expectation for $L=100 L_{G}$ in our numerical calculations; in particular, the discrete eigenvalues of the finite geometry become very dense and hard to separate. Thus we are able to reproduce the dispersion relation (1) for the case of independent interfaces.

\section{A. Near-tip behavior}

The tip of a crack is typically subject to divergencies. For example, the stress field behaves like $\sigma \sim r^{-1 / 2}$, where $r$ is the distance from the tip. From Eq. (10) it follows readily that the different contributions to chemical potential behave like

$$
\begin{gathered}
\mu_{\text {sur }} \sim y^{\prime \prime}(x), \\
\mu_{e l s} \sim\left(L^{2}-x^{2}\right)^{-3 / 2} y(x), \\
\mu_{e l u} \sim\left(L^{2}-x^{2}\right)^{-1 / 2} \mathcal{P} \int_{-L}^{L} y^{\prime}(t) \frac{\sqrt{L^{2}-t^{2}}}{t-x} d t .
\end{gathered}
$$

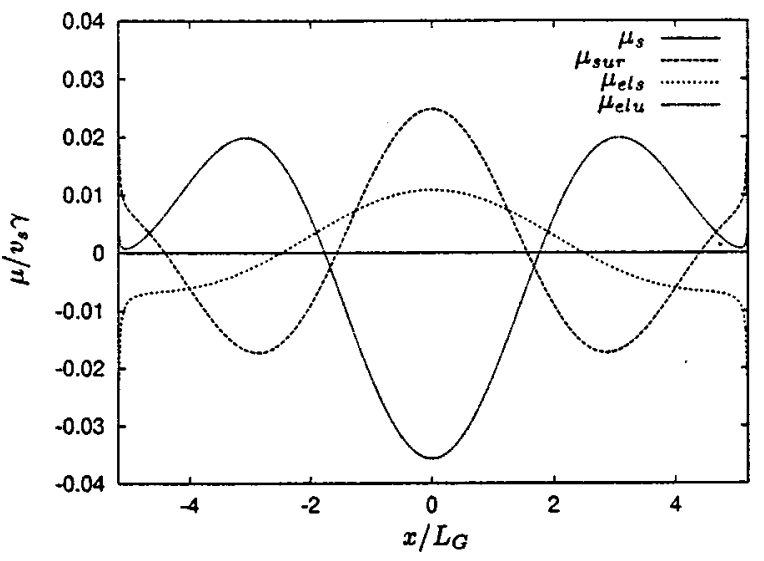

FIG. 8. Contributions to the chemical potential of a critical crack with $L=5.187 L_{G}, \lambda=0, M=400$.

For $L=L_{c}$, they must cancel each other everywhere on the crack: $\mu_{s}+\mu_{e l s}+\mu_{e l u} \equiv 0$. It is difficult to justify this cancellation for the Fourier representation (12) of the previous section because of strong oscillations near the crack tip, though it holds nicely in the center region $x \approx 0$. The reason is that though the full Fourier series with summation cutoff $K \rightarrow \infty$ is complete, $K<\infty$ always implies that nonvanishing values of $y^{\prime \prime}( \pm L)$ can only be achieved asymptotically. With the real space representation we do not suffer from this problem, and indeed this cancellation seems to be verified, see Fig. 8.

In Ref. [10] the guess was made that the divergent contributions of $\mu_{e l u}$ and $\mu_{e l s}$ cancel each other at the crack tip. This is equivalent to a vanishing of the total mode II stress intensity factor $K_{I I}^{(t o t)}$. The expression for this value has been derived in Ref. [4] for the case of a slightly wavy crack. To first order in $y$ it is given by [5]

$$
K_{I I}^{(t o t)}=K_{I I}(L)+\sqrt{L \pi} P y^{\prime}(L) / 2
$$

It reflects exactly the decomposition of the elastic field into a stabilizing and unstabilizing part as used in the first section of this paper. The divergent component of the tangential stress along the surfaces of a wavy crack is given by

$$
\sigma_{\tau \tau}^{(\sin g)}=-\frac{2 K_{I I}^{(t o t)}}{(2 \pi r)^{1 / 2}}
$$

in the close vicinity of the tip. One can easily check that the second contribution to $K_{I I}^{(t o t)}$ produces exactly the divergent part of the stress field (4) and therefore of the chemical potential $\mu_{e l s}$ in Eq. (23).

It is clear from the representation (22)-(24) above that a cancellation of the divergencies of $\mu_{e l u}$ and $\mu_{e l s}$ is equivalent to a finite crack curvature at the tips and, from Eq. (26), also to the vanishing of the stress intensity factor $K_{I I}^{(t o t)}$. The latter criterion is widely discussed in literature as a criterion for the direction of crack propagation, referred to as the "criterion of local symmetry.' It states that a crack propagates in 
the direction of maximum energy release, maximum hoop stress or stationary Sih energy density factor (see references in [4] for a survey).

Surprisingly the calculations show that this criterion is not fulfilled here. It turns out that a divergent curvature contribution remains. Calculations with extreme accuracy raise the claim that $y(r) \sim r+r^{\gamma}$ at the tips of a critical crack with $L$ $=5.187 L_{G}$, with $\gamma \approx 1.5$ (see Fig. 9). For these calculations it is necessary to concentrate as many grid points as possible in the vicinity of the tips where the shape functions vary crucially, whereas a moderate accuracy suffices in the middle part.

The reason for this unexpected behavior is that the $K_{I I}^{(t o t)}=0$ criterion maximizes only the release of elastic energy, and does not take surface energy into account; it simply compares different directions of elongation and leaves behind the crack as the track of the tip. In our case, the elongation is completely forbidden, but we allow for a deformation of the already existing crack. Therefore completely different crack shapes are compared to each other to minimize the total energy. For fast crack growth the deformation can be neglected since it is driven by the slow surface diffusion, but it is still an unanswered question how $K_{I I}^{(t o t)}=0$ and $\mu=$ const can be reconciled for a slow motion of the tip, when both processes, crack propagation and Grinfeld instability, are present.

\section{B. Grinfeld instability on incoherent crack surfaces}

In this subsection we give up the restriction of parallel crack surfaces. Both surfaces are described by independent shape functions $y_{u}(x)$ and $y_{l}(x)$, provided that $y_{u / l}( \pm L)$ $=0$. Of course it must be assured that the two branches of the crack do not overlap, i.e., $y_{u}(x)>y_{l}(x)$. However, a small opening of the crack is present due to the applied loading even in the case of a straight crack. Consequently a small perturbation with a sufficient small amplitude does not lead to an intersection.

The dynamics of both interfaces is again described by surface diffusion $\dot{y}_{u / l}=\mp D \nabla^{2} \mu_{u / l}$ and the boundary conditions

$$
\mu_{u}( \pm L)=\mu_{l}( \pm L), \quad y_{u / l}( \pm L)=0
$$

and also flux conservation

$$
\mu_{u}^{\prime}( \pm L)=-\mu_{l}^{\prime}( \pm L)
$$

This implies the mass conservation law

$$
\int_{-L}^{L}\left[y_{u}(x)-y_{l}(x)\right] d x=\text { const }
$$

which is trivially fulfilled for parallel crack surfaces.

For the moment we restrict our considerations to antiparallel crack surfaces $y_{u(x)}=-y_{l(x)}=y(x)$. In this case $\mu_{u}(x)$ $=\mu_{l}(x)=\mu(x)$ holds everywhere and thus the condition (27) is satisfied automatically. Equation (28) requires the disappearance of the tip flux $\mu^{\prime}( \pm L)=0$.
We analyze this problem in the same way as before. It turns out that the spectrum is very similar to that of coherent crack surfaces. The main result is that the critical length is slightly bigger in this arrangement: $L_{c}^{(a n t i)}=5.212 L_{G}$. It means that during a slow elongation of the crack the parallel modes will become visible first.

It is now easy to generalize this statement to arbitrary crack surfaces $y_{u / l}$. Since we are basically interested in the threshold of instability, we use again the energetic argument of the last section. To understand this behavior, we decompose the shape functions into a parallel and an antiparallel contribution:

$$
y_{p}:=\left(y_{u}+y_{l}\right) / 2, \quad y_{a}:=\left(y_{u}-y_{l}\right) / 2
$$

From Eqs. (2) and (A16) it follows readily that the chemical potential decomposes similarly,

$$
\begin{gathered}
\mu_{u}(x)=\mu_{p}(x)+\mu_{a}(x), \\
\mu_{l}(x)=-\mu_{p}(x)+\mu_{a}(x),
\end{gathered}
$$

with

$$
\mu_{p}(x)=v_{s}\left[\frac { 1 - \nu ^ { 2 } } { 2 E } \left\{-\frac{4 P^{2} L^{2}}{\left(L^{2}-x^{2}\right)^{3 / 2}} y_{p}(x)\right.\right.
$$

$$
\begin{gathered}
\left.-\frac{4 P^{2}}{\pi\left(L^{2}-x^{2}\right)^{1 / 2}} \mathcal{P} \int_{-L}^{L} \frac{y_{p}^{\prime}(t)}{t-x} \sqrt{L^{2}-t^{2}} d t\right\} \\
\left.+\alpha y_{p}^{\prime \prime}(x)\right], \\
\mu_{a}(x)=v_{s}\left[f_{s 0}+\frac{1-\nu^{2}}{2 E}\left\{P^{2}-\frac{4 P^{2} L^{2}}{\left(L^{2}-x^{2}\right)^{3 / 2}} y_{a}(x)\right.\right. \\
\left.\left.-\frac{4 P^{2}}{\pi} \mathcal{P} \int_{-L}^{L} \frac{y_{a}^{\prime}(t)}{t-x} d t\right\}+\alpha y_{a}^{\prime \prime}(x)\right] .
\end{gathered}
$$

The crucial observation is that the total energy

$$
U=\frac{1}{2 v_{s}}\left(-\int_{-L}^{L} \mu_{u}(x) y_{u}(x) d x+\int_{-L}^{L} \mu_{l}(x) y_{l}(x) d x\right)
$$

becomes diagonal in this representation:

$$
U=-\frac{1}{v_{s}} \int_{-L}^{L}\left(\mu_{p} y_{p}+\mu_{a} y_{a}\right) d x .
$$

Thus the parallel and antiparallel configurations are the " "principal axes" of the energy ellipsoid. We can therefore conclude that the parallel arrangement of surfaces indeed gives the most unstable configuration. 


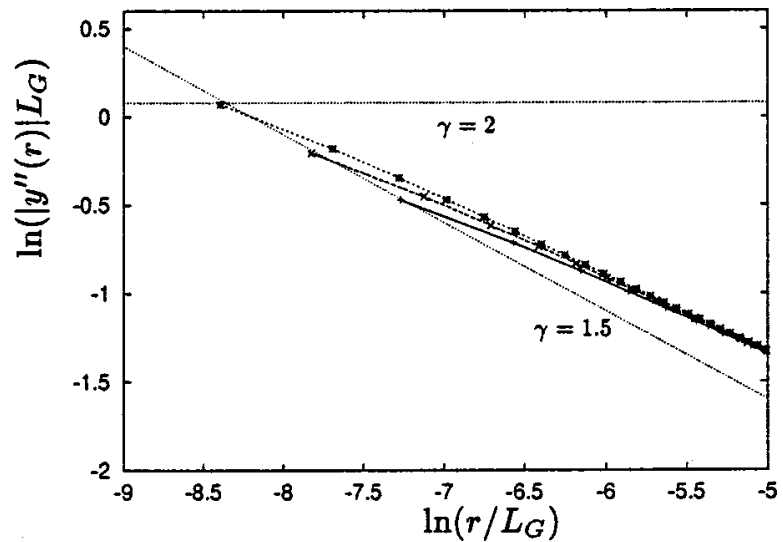

FIG. 9. Logarithmic plot of the curvature near the tips of a critical crack, $L=5.187 L_{G}, \lambda=0$. All curves correspond to the same number of grid points but different spatial distributions. The solid line possesses the least grid point density near the tips, the dotted one the highest. With increasing accuracy the coefficient $\gamma$ comes closer and closer towards 1.5 .

\section{CRACKS UNDER GENERALIZED MODE I LOADING}

So far in all situations a pure uniaxial stress $P>0$, perpendicular to the crack, has been exerted on the solid. Now we add an additional stress component $P_{x}$ at infinity parallel to the crack ( $x$ direction). In the framework of a linear theory of elasticity this homogeneous field is simply added to the former field. Since it gives a new contribution to the shear traction of a wavy crack, the elastic energy is modified. We declare $P_{x}$ to be positive for a tensile stress and introduce the dimensionless parameter $\beta=P_{x} / P$. One easily derives

$$
\begin{gathered}
U_{e l s}(\beta)=(1-\beta) U_{e l s}(\beta=0), \\
U_{e l u}(\beta)=(1-\beta)^{2} U_{e l u}(\beta=0) .
\end{gathered}
$$

Hence the critical length becomes a function of $\beta$ as well; this dependence is plotted in Fig. 10. For $\beta=0$ we retain the former result $L_{c}=5.18 L_{G}$.

The most interesting range is $0<\beta<1$ : The pure tensile loading perpendicular to the crack causes a tangential stress $\sigma_{x x}=-P$ along a straight crack. The additional loading $P_{x}$ $>0$ reduces this value to $\sigma_{0}=\sigma_{x x}=-P+P_{x}<0$ and thus hampers the evolution of the Grinfeld instability. Therefore the critical length increases in comparison to $\beta=0$. For $\beta$ $\rightarrow 1$ the nonhydrostaticity vanishes completely and the Grinfeld instability cannot occur. Consequently the critical length diverges. In other words, the change of the elastic energy during redistribution of matter is at least of order $O\left(y^{4}\right)$ in the deviation from the straight line $y=0$. This result has already been derived in Ref. [16]. The operator of the chemical potential therefore consists (to the lowest nonvanishing order) only of the local curvature operator $\mu_{s} \sim v_{s} \alpha \kappa$ that has eigenfunctions $y_{k} \sim \sin (\pi k x / L)$. Thus energy becomes diagonal in this Fourier representation. We just remark that these eigenfunctions are completely different from the ones obtained above. The most important fact is that they do not exhibit a divergency of curvature near the tips.

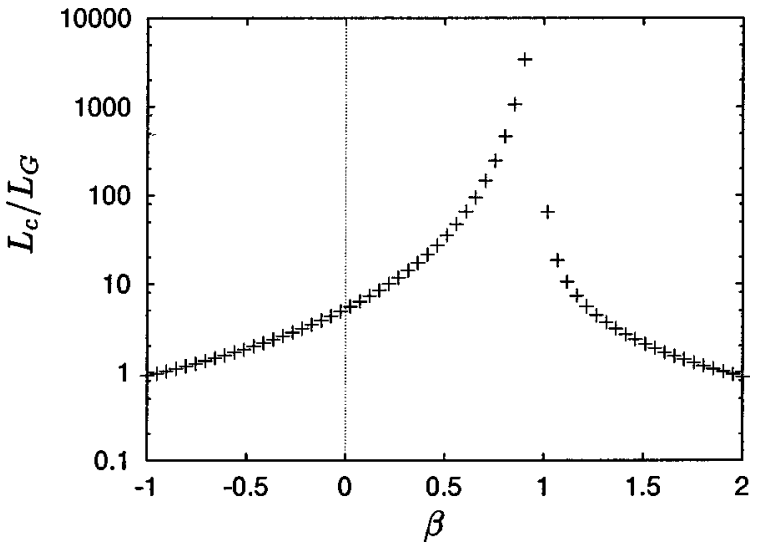

FIG. 10. Dependence of the critical length on the loading ratio $\beta=P_{x} / P$.

For $\beta<0$ the compressive surface stress is even increased and the critical length becomes smaller.

The case $\beta>1$ means that the horizontal stress becomes bigger than the vertical loading. Generally the elastic energy release $d U_{e l} / d L \sim K_{I}^{2}+K_{I I}^{2}$ during crack relaxation is maximized if the crack orients perpendicular to the direction of the highest stress. A vertical orientation of the crack is forbidden by the boundary conditions $y( \pm L)=0$; but by taking on a wavy shape the crack tries to mimic this optimal shape. This tendency becomes stronger with higher values of $P_{x}$. Therefore $L_{c} \rightarrow 0$ for $\beta \rightarrow \infty$.

\section{SUMMARY AND DISCUSSION}

We calculated expressions for the total energy of a wavy crack that is subjected to a mode I loading. It turned out that, for a loading perpendicular to the crack, this energy becomes smaller, when a critical crack length, $L_{c}=5.18 L_{G}$, is exceeded. Then the Grinfeld instability can develop and a formation of deep grooves along the crack surfaces becomes possible. This deformation is due to a redistribution of matter and not due to instabilities of the moving crack tip. We determined the threshold of instability either using a static, energetic description, either through the early dynamical evolution via surface diffusion or melting and recrystallization.

We generalized the loading condition by adding a stress component parallel to the crack; this modifies the threshold of instability in a nontrivial way. Furthermore the situation of independent crack surfaces has been studied, with the result that the parallel configuration is the most unstable one.

It turned out that the eigenfunctions of the equations of motion exhibit a singular tip curvature. This corresponds to a nonvanishing stress intensity factor $K_{I I}^{(t o t)}$. The principle of local symmetry states that during crack propagation processes the direction of extension is oriented such that $K_{I I}^{(t o t)}$ $=0$ is satisfied. In our model the tip positions are fixed and we studied only the slower surface kinetics.

The most important outstanding problem is the combination of crack propagation and Grinfeld instability. We mention that the start of the Grinfeld instability is beyond the Griffith threshold, and therefore the deformations naturally happen only in the regime of fast propagating cracks. There- 
fore we briefly discuss dynamical aspects of the Grinfeld instability when the tip velocity is not assumed to be small. However, the stress distribution on the crack surface remains qualitatively the same as in the static case, apart from some factors that depend on the tip velocity $V[1]$. Therefore our predictions that were performed for a static crack remain qualitatively correct even in the limit of fast crack extension.

Far from the tips of a long, static crack with $L \gg L_{G}$, the usual Grinfeld spectrum (1) is valid in a local sense. For a fast propagating crack it is slightly modified due to inertial effects. In the laboratory frame of reference it can be written as

$$
\lambda=D v_{s} k^{2}\left(\frac{4 \alpha q}{\pi L_{G}}|k|-\alpha k^{2}\right),
$$

where $q$ is a dimensionless function that depends weakly on $V / V_{R}$ with the Rayleigh speed $V_{R}$ and the Poisson ratio $\nu$.

One can expect that the linear instability described by the local dispersion relation (37) should be only convective in the frame of reference of the moving tip due to its slow development compared to the fast tip motion. Indeed the most unstable mode corresponds to the values $k \sim q / L_{G}$ and $\lambda=\lambda_{u} \sim D q^{4} / L_{G}^{4}$. In the moving frame of reference, $\lambda$ should be replaced by $(\lambda-i V k)$ that contains a convective contribution of the order $\lambda_{c} \sim q V / L_{G}$. The ratio $\lambda_{u} / \lambda_{c}$ $\sim q^{3} D /\left(L_{G}^{3} V\right)$ is expected to be small if the velocity $V$ is of order of the Rayleigh speed. This corresponds only to the convective instability [17]. In this sense, the tip motion itself is insensitive to the development of the instability behind the tip. Nevertheless, the drastic acceleration of the instability and the refining of the length scale in the nonlinear regime [9] make it still conceivable that also the tip motion could be affected by the instability.

We also remark that crack growth by tip propagation is more generally hampered not by surface energy $\alpha$ but by the so-called fracture energy $\Gamma$ that represents the resistance of the material to crack advance [1]. This material parameter is usually bigger than the surface energy, and therefore for some materials it is conceivable that the threshold of Grinfeld instability (which still depends on the smaller surface energy $\alpha$ ) and crack propagation are quite close to each other. This increases the chance that the relatively slow tip motion is yet influenced by the Grinfeld instability.

The main problem in observing the phenomenon of the Grinfeld instability is that it can be obscured by the fast crack propagation for crack lengths $L>L_{G}$. It arises from the fact that the crack length (or the applied tension) is the only tunable parameter, and the two effects cannot be separated. However, this dilemma can be solved by another experiment that has already been successfully used in the past [18] and is sketched in Fig. 11. The observed instabilities of the crack shape were interpreted in the framework of the principle of local symmetry and not as a result of the Grinfeld instability. It turned out that at the threshold of instability the energy of a wavy crack is already lower than of a straight crack.

A long thin glass strip is pulled from a hot region (heater) to a cold one (water bath) at a slow and constant velocity $V$.

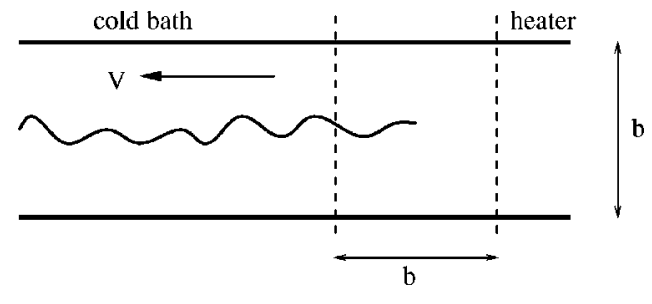

FIG. 11. Suggestion of an experiment to observe the Grinfeld instability on the surfaces of a slowly propagating crack.

With a suitable choice of the control parameters, the temperature decrease $\Delta T$ between the hot and cold region and the distance $b$ between them, a stationary semi-infinite crack growth with velocity $V$ becomes possible. All elastic strains arise here from a thermal gradient $\nabla T \sim \Delta T / b$; no external tension is applied here. For simplicity we choose the strip width also to be of order $b$; in other words, $b$ is the only relevant length scale in the problem. Far from the tip in the cold and hot regions the material is completely relaxed. In the transition region the characteristic stresses are $\sigma$ $\sim E \alpha_{T} \Delta T$ ( $\alpha_{T}$ is the coefficient of thermal expansion). They result in a stress intensity factor $K \sim \sigma b^{1 / 2}$. By Irwin's theorem an advance of the crack tip by the length $d s$ reduces the elastic energy by $\delta W_{e l} \sim K^{2} / E d s$. It is accompanied by an increase of the surface energy by $\delta W_{s} \sim \alpha d s$. Near the Griffith threshold $W_{e l} \sim W_{s}$ the propagation velocity is arbitrarily small; it corresponds to the temperature difference $(\Delta T)^{2}$ $\sim \alpha /\left(b E \alpha_{T}^{2}\right)$. On the other hand, the characteristic wavelength of the Grinfeld instability is of the order $L_{G}$ $\sim E \alpha / \sigma^{2} \sim b$, in agreement with the above statement that all length scales are of order $b$. By this means it should be possible to observe the Grinfeld instability in a system of a slowly propagating or even stationary crack.

Alternatively, it could also be possible to observe the Grinfeld instability in the following way: As it was shown in Sec. IV, it is possible to alter the critical length by applying an additional stress in longitudinal direction together with the perpendicular loading. This allows us, for example, to keep the crack exactly at the Griffith threshold, but still exceeding the critical length for the Grinfeld instability. It is a completely unanswered question how the criterion of local symmetry and the contradicting result of divergent tip curvature come together in this regime. Further analysis of this problem is required in the future.

Another unclear point is the exact behavior near the crack tips. Basically we analyzed long wave perturbations of the crack shape, where the wavelength is of the order of the length of the crack itself. However, in our model of a mathematical crack we observed a divergence of the curvature near the crack tips. This stimulates the suspicion of the importance of this region. In particular, one can speculate that perturbations of a rounded tip, with a wavelength of the order of the tip radius, may lead to important new features, including a tip splitting as a small scale instability. This requires the introduction of a new degree of freedom, the radius of curvature of the blunt tip. We stress that even if surface diffusion is slow on the macroscopic scale, it can still be very efficient on the microscopic scale in the vicinity of 


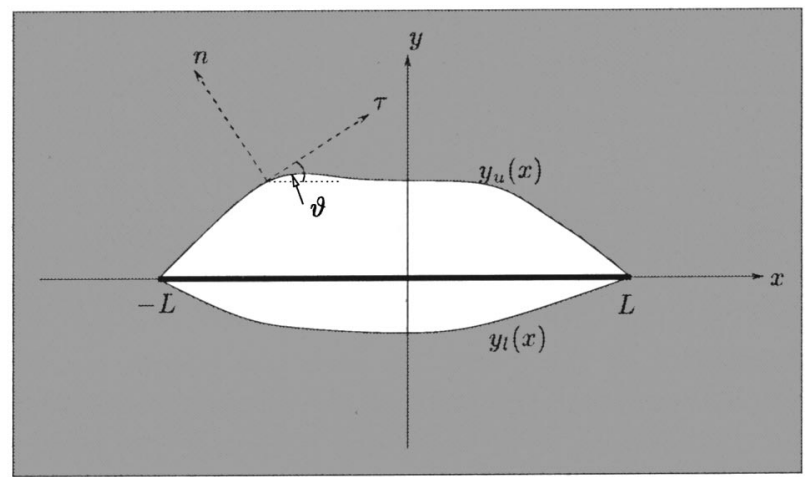

the tip. A similar idea has been introduced by Langer [19] to describe the plastic flow in the vicinity of a crack tip, where he used an elliptic crack to incorporate the tip curvature. A future goal is to include such a description into our model. However, in the brittle theory of fracture a mechanism to regularize the tip curvature is not present; consequently further research is necessary in this direction to clear up this point.

\section{APPENDIX A: SLIGHTLY CURVED CRACKS}

The aim of this section is to derive expressions for the stress field around a curved crack that is subjected to a mode I loading. We generalize the results from [4] to describe the case of nonparallel crack surfaces as depicted in Fig. 12.

Since we deal with a two-dimensional plane strain situation, Muskhelishvili's analytic function method can be used [20]. The whole information is contained in two analytic functions $\varphi(z)$ and $\chi(z)$. Stresses can be expressed as follows:

$$
\begin{gathered}
\sigma_{x x}+\sigma_{y y}=4 \operatorname{Re}[\varphi(z)], \\
\sigma_{y y}-\sigma_{x x}+2 i \sigma_{x y}=2\left[\bar{z} \varphi^{\prime}(z)+\chi(z)\right] .
\end{gathered}
$$

As usual we reduce the elastic problem to the case of vanishing stresses at infinity and given loadings $\left[\sigma_{n n}\right]_{u / l}$ and $\left[\sigma_{n \tau}\right]_{u / l}$ along upper and lower crack surfaces $y_{u / l}(x)$. For convenience we introduce the function

$$
\psi(z):=\bar{\varphi}(z)+z \overline{\varphi^{\prime}}(z)+\bar{\chi}(z)
$$

where we used the notation $\bar{\varphi}(z):=\overline{\varphi(\bar{z})}$. The boundary values can be expressed by

$$
\begin{aligned}
\sigma_{n n}-i \sigma_{n \tau}= & \varphi(z)+\overline{\varphi(z)} \\
& +e^{-2 i \vartheta}\left[(z-\bar{z}) \overline{\varphi^{\prime}(z)}+\psi(\bar{z})-\overline{\varphi(z)}\right]
\end{aligned}
$$

As in Ref. [4] we allow only small deviations from the straight crack, $\left|y_{u / l}(x)\right| \ll L$, and perform a perturbation
FIG. 12. Geometry of the curved crack. The branch cut of the analytic functions is indicated by the thick line. analysis. Therefore we expand $\varphi$ and $\psi$ with respect to the "'smallness parameter' $y_{u / l}$ and retain only first order contributions,

$$
\varphi(z)=F_{0}(z)+F_{1}(z), \quad \psi(z)=W_{0}(z)+W_{1}(z)
$$

where the functions $F_{i}(z)$ and $W_{i}(z)$ are everywhere analytic apart from the straight crack line $[-L, L]$ and are of order $i$ in the expansion parameter $y_{u / l}$. We introduce the common abbreviation

$$
\varphi^{ \pm}(x)=\lim _{\varepsilon \rightarrow 0 \pm} \varphi(x+i \varepsilon) .
$$

To first order one can write

$$
\begin{aligned}
& \varphi\left(x+i y_{u}(x)\right)=F_{0}^{+}(x)+i y_{u}(x) F_{0}^{+\prime}(x)+F_{1}^{+}(x), \\
& \varphi\left(x+i y_{l}(x)\right)=F_{0}^{-}(x)+i y_{l}(x) F_{0}^{-\prime}(x)+F_{1}^{-}(x),
\end{aligned}
$$

and similarly for $\psi$. Noting that $\exp (-2 i \vartheta)=1-2 i y_{u / l}^{\prime}(x)$ to first order, the boundary values on the straight cut, Eq. (A3), become

$$
\begin{aligned}
{\left[\sigma_{n n}-i \sigma_{n \tau}\right]_{u / l}=} & F_{0}^{ \pm}(x)+i y_{u / l}(x) F_{0}^{ \pm \prime}(x)+F_{1}^{ \pm}(x) \\
& +2 i y_{u / l}(x) \overline{F_{0}^{ \pm \prime}(x)}+W_{0}^{\mp}(x) \\
& -i y_{u / l}(x) W_{0}^{\mp \prime}(x)+W_{1}^{\mp}(x) \\
& -2 i y_{u / l}^{\prime}(x)\left(W_{0}^{\mp}(x)-\overline{F_{0}^{ \pm}(x)}\right) .
\end{aligned}
$$

Thus separating zero and first order give

$$
\begin{gathered}
{\left[\sigma_{n n}^{(0)}-i \sigma_{n \tau}^{(0)}\right]_{u / l}=F_{0}^{ \pm}(x)+W_{0}^{\mp}(x),} \\
F_{1}^{ \pm}(x)+W_{1}^{\mp}(x)=\left[\sigma_{n n}^{(1)}-i \sigma_{n \tau}^{(1)}\right]_{u / l}-\left(i y_{u / l}(x)\left[F_{0}^{ \pm}(x)+W_{0}^{\mp}\right]^{\prime}\right. \\
\left.+2 i\left\{y_{u / l}(x)\left[\overline{F_{0}^{ \pm}(x)}-W_{0}^{\mp}(x)\right]\right\}^{\prime}\right) .
\end{gathered}
$$

Here $\left[\sigma^{(i)}\right]_{u / l}$ are the contributions of order $i$ with respect to $y_{u / l}$ to the prescribed stresses $[\sigma]_{u / l}$. The boundary values of $\left[F_{0}(x)+W_{0}(x)\right]$ and $\left[F_{0}(x)-W_{0}(x)\right]$ are given by 


$$
\begin{gathered}
{\left[F_{0}(x)+W_{0}(x)\right]^{+}+\left[F_{0}(x)+W_{0}(x)\right]^{-}} \\
=\left[\sigma_{n n}^{(0)}-i \sigma_{n \tau}^{(0)}\right]_{u}+\left[\sigma_{n n}^{(0)}-i \sigma_{n \tau}^{(0)}\right]_{l}, \\
{\left[F_{0}(x)-W_{0}(x)\right]^{+}-\left[F_{0}(x)-W_{0}(x)\right]^{-}} \\
\quad=\left[\sigma_{n n}^{(0)}-i \sigma_{n \tau}^{(0)}\right]_{u}-\left[\sigma_{n n}^{(0)}-i \sigma_{n \tau}^{(0)}\right]_{l} .
\end{gathered}
$$

Using the formula of Muskhelishvili [20],

$$
\begin{aligned}
F_{0}(z)= & \frac{1}{4 \pi(z+L)^{1 / 2}(z-L)^{1 / 2}} \\
& \times \int_{-L}^{L} \frac{\left[\sigma_{n n}^{(0)}-\sigma_{n \tau}^{(0)}\right]_{u}+\left[\sigma_{n n}^{(0)}-\sigma_{n \tau}^{(0)}\right]_{l}}{x-z}\left(L^{2}-x^{2}\right)^{1 / 2} d x \\
& +\frac{1}{4 \pi i} \int_{-L}^{L} \frac{\left[\sigma_{n n}^{(0)}-i \sigma_{n \tau}^{(0)}\right]_{u}-\left[\sigma_{n n}^{(0)}-i \sigma_{n \tau}^{(0)}\right]_{l}}{x-z} d x
\end{aligned}
$$

$$
\begin{aligned}
W_{0}(z)= & \frac{1}{4 \pi(z+L)^{1 / 2}(z-L)^{1 / 2}} \\
& \times \int_{-L}^{L} \frac{\left[\sigma_{n n}^{(0)}-\sigma_{n \tau}^{(0)}\right]_{u}+\left[\sigma_{n n}^{(0)}-\sigma_{n \tau}^{(0)}\right]_{l}}{x-z}\left(L^{2}-x^{2}\right)^{1 / 2} d x \\
& -\frac{1}{4 \pi i} \int_{-L}^{L} \frac{\left[\sigma_{n n}^{(0)}-i \sigma_{n \tau}^{(0)}\right]_{u}-\left[\sigma_{n n}^{(0)}-i \sigma_{n \tau}^{(0)}\right]_{l}}{x-z} d x .
\end{aligned}
$$

The second integral appearing in both of these two formulas does not exhibit the usual square root singularity and becomes only relevant in the case of nonparallel crack surfaces.

Basically we are interested in the tangential component of the stress tensor,

$$
\sigma_{\tau \tau}=\operatorname{Re}\left(2 \varphi(z)-e^{-2 i \vartheta}\left[(z-\bar{z}) \overline{\varphi^{\prime}(z)}+\psi(\bar{z})-\overline{\varphi(z)}\right]\right)
$$

Evaluating the limiting values of Eqs. (A6) and (A7), we obtain to zeroth order

$$
\left[\sigma_{\tau \tau}^{(0)}\right]_{u / l}=\left[s_{\tau \tau}^{(0)}\right]_{u / l},
$$

where we have defined the function

$$
\begin{aligned}
{\left[s_{\tau \tau}^{(i)}\right]_{u / l}=} & {\left[\sigma_{n n}^{(i)}\right]_{u / l} \mp \frac{1}{\pi\left(L^{2}-x^{2}\right)^{1 / 2}} } \\
& \times \mathcal{P} \int_{-L}^{L} \frac{\left[\sigma_{n \tau}^{(i)}\right]_{u}+\left[\sigma_{n \tau}^{(i)}\right]_{l}}{t-x} \sqrt{L^{2}-t^{2}} d t \\
& +\frac{1}{\pi} \mathcal{P} \int_{-L}^{L} \frac{-\left[\sigma_{n \tau}^{(i)}\right]_{u}+\left[\sigma_{n \tau}^{(i)}\right]_{l}}{t-x} d t
\end{aligned}
$$

In the same way one can calculate the first order contribution, but the result is quite lengthy. In our case we have a uniform mode I loading at infinity, i.e., up to the first order

$$
\begin{gathered}
{\left[\sigma_{n n}\right]_{u / l}=\left[\sigma_{n n}^{(0)}\right]_{u / l}+\left[\sigma_{n n}^{(1)}\right]_{u / l}=-P,} \\
{\left[\sigma_{n \tau}\right]_{u / l}=\left[\sigma_{n \tau}^{(0)}\right]_{u / l}+\left[\sigma_{n \tau}^{(1)}\right]_{u / l}=-P y_{u / l}^{\prime}(x) .}
\end{gathered}
$$

Thus the normal traction is constant and the shear stress has only a first order contribution. This simplifies the expressions and one finally obtains, after some simple transformations, $\left[\sigma_{\tau \tau}^{(0)}\right]_{u / l}=-P$, as expected for the straight crack, and

$$
\left[\sigma_{\tau \tau}^{(1)}\right]_{u / l}=\left[s_{\tau \tau}^{(1)}\right]_{u / l} \pm \frac{2 P L^{2}}{\left(L^{2}-x^{2}\right)^{3 / 2}} y_{u / l}(x)
$$

The final result is therefore as follows: A curved crack loaded by $\sigma_{y y}^{\infty}=P$ and $\sigma_{i j}^{\infty}=0$ for all other components at infinity exhibits total stresses at the interfaces of the crack given by

$$
\begin{gathered}
{\left[\sigma_{n n}^{(t o t)}\right]_{u / l}=0,} \\
{\left[\sigma_{n \tau}^{(t o t)}\right]_{u / l}=0,} \\
{\left[\sigma_{\tau \tau}^{(t o t)}\right]_{u / l}=\left[\sigma_{\tau \tau}^{(0)}\right]_{u / l}+\left[\sigma_{\tau \tau}^{(1)}\right]_{u / l}}
\end{gathered}
$$

to first order, using the expressions (A9)-(A13). Here we have already added the homogeneous stress caused by the mode I loading at infinity.

\section{APPENDIX B: EQUIVALENCE OF ENERGY REPRESENTATIONS}

To show the equivalence of the different approaches to calculate the total energy of a wavy crack (8) and the direct integration of the chemical potential (10), it is sufficient to derive the chemical potential from the first approach

$$
\left[\mu_{s}(x)\right]_{u / l}=\mp \frac{v_{s}}{2} \frac{\delta U}{\delta y(x)}
$$

and to compare it with the original expression (10). Since this is straightforward for $U_{s}$ and $U_{e l s}$, we restrict our con- 
siderations to $U_{\text {elu }}$ and give only some brief hints for deduction. Using Eqs. (7) and (8), we obtain

$$
\begin{aligned}
U_{e l u}= & -\frac{1-\nu^{2}}{E} \frac{2 P^{2}}{\pi} \int_{L^{\prime}=-L}^{L} \int_{x=-L}^{L^{\prime}} \int_{t=-L}^{L^{\prime}} \frac{1}{L+L^{\prime}} \sqrt{\frac{L+x}{L^{\prime}-x}} \\
& \times \sqrt{\frac{L+t}{L^{\prime}-t} y^{\prime}(x) y^{\prime}(t) d t d x d L^{\prime} .}
\end{aligned}
$$

The main idea is to interpret the triple integral as volume integral. Rewriting it as

$$
\begin{aligned}
& \int_{L^{\prime}=-L}^{L} \int_{x=-L}^{L^{\prime}} \int_{t=-L}^{L^{\prime}} \cdots d t d x d L^{\prime} \\
& \quad=\int_{t=-L}^{L} \int_{x=-L}^{L} \int_{L^{\prime}=\max (t, x)}^{L} \cdots d L^{\prime} d x d t,
\end{aligned}
$$

allows us to perform the explicit calculation of the innermost integral. For simplicity we ignore the difficulties arising from the exchange of the integration order. In fact, they are responsible for the appearance of the principal value integrals, but we treat all integrals as (divergent) ordinary integrals, because we are basically interested in a structural agreement of the expressions. However, all calculations can easily be extended to overcome this limitation.

We conclude

$$
\begin{aligned}
U_{e l u}[y+\delta y]-U_{e l u}[y] & \\
= & \frac{2 P^{2}\left(1-\nu^{2}\right)}{E \pi} \int_{t=-L}^{L} \delta y^{\prime}(t) \int_{x=-L}^{L} 2 y^{\prime}(x) \\
& \times\left\{\ln \left(\frac{L^{2}-x t-\sqrt{\left(L^{2}-t^{2}\right)\left(L^{2}-x^{2}\right)}}{L}\right)-\ln |x-t|\right\} d x d t,
\end{aligned}
$$

where only first order contributions have been taken into account. Another integration by parts [notice that $y( \pm L)$ $=0]$ and further algebraic manipulations lead to

$$
\begin{aligned}
U_{e l u}[y+\delta y]-U_{e l u}[y]= & -\frac{4 P^{2}\left(1-\nu^{2}\right)}{\pi E} \int_{t=-L}^{L} \delta y(t) \\
& \times \int_{x=-L}^{L} \frac{y^{\prime}(x)}{t-x} \sqrt{\frac{L^{2}-x^{2}}{L^{2}-t^{2}}} d x d t .
\end{aligned}
$$

Now the functional derivative can be immediately read:

$$
\left[\mu_{e l u}\right]_{u / l}= \pm \frac{2 v_{s} P^{2}\left(1-\nu^{2}\right)}{\pi E \sqrt{L^{2}-t^{2}}} \int_{x=-L}^{L} \frac{y^{\prime}(x)}{t-x} \sqrt{L^{2}-x^{2}} d x
$$

It matches the third term in Eq. (10), of course apart from the principal value. This completes the proof.

(1999).

[10] E.A. Brener and V.I. Marchenko, Phys. Rev. Lett. 81, 5141 (1998).

[11] C. Herring, J. Appl. Phys. 21, 437 (1950); P. Nozières, J. Phys. I 3, 681 (1993).

[12] J.R. Rice, in Fracture: An Advanced Treatise, edited by H. Liebowitz (Academic, New York, 1968), Vol. 2, Chap. 3, pp. 191-311.

[13] G.R. Irwin, J. Appl. Mech. 24, 361 (1957).

[14] J.H. Wilkinson, The Algebraic Eigenvalue Problem (Oxford University Press, London, 1988).

[15] W.H. Press et al., Numerical Recipes in C (Cambridge University Press, Cambridge, England, 1996).

[16] A. Buchel and J. Sethna, Phys. Rev. E 55, 7669 (1997).

[17] E.M. Lifshitz and L.P. Pitaevskii, Physical Kinetics (Pergamon Press, Oxford, 1981).

[18] A. Yuse and M. Sano, Nature (London) 362, 329 (1993); M. Adda-Bedia and Y. Pomeau, Phys. Rev. E 52, 4105 (1995).

[19] J.S. Langer, Phys. Rev. E 62, 1351 (2000).

[20] N.I. Muskhelishvili, Some Basic Problems on the Mathematical Theory of Elasticity (Noordhoff, Groningen, 1952). 